\title{
Brief Paper: \\ Evaluation of Subtractive Clustering based Adaptive Neuro-Fuzzy Inference System with Fuzzy C-Means based ANFIS System in Diagnosis of Alzheimer
}

\author{
Haneet Kour ${ }^{*}$, Jatinder Manhas², Vinod Sharma ${ }^{3}$
}

\begin{abstract}
Machine learning techniques have been applied in almost all the domains of human life to aid and enhance the problem solving capabilities of the system. The field of medical science has improved to a greater extent with the advent and application of these techniques. Efficient expert systems using various soft computing techniques like artificial neural network, Fuzzy Logic, Genetic algorithm, Hybrid system, etc. are being developed to equip medical practitioner with better and effective diagnosing capabilities. In this paper, a comparative study to evaluate the predictive performance of subtractive clustering based ANFIS hybrid system (SCANFIS) with Fuzzy C-Means (FCM) based ANFIS system (FCMANFIS) for Alzheimer disease (AD) has been taken. To evaluate the performance of these two systems, three parameters i.e. root mean square error (RMSE), prediction accuracy and precision are implemented. Experimental results demonstrated that the FCMANFIS model produce better results when compared to SCANFIS model in predictive analysis of Alzheimer disease (AD).
\end{abstract}

Key Words: AD, ANFIS, Fuzzy C-Means, Subtractive Clustering.

\section{INTRODUCTION}

Alzheimer disease (AD) is emerging as the most leading form of dementia interfering severely with daily tasks of the patient. As per 2017 Alzheimer's Statistics: about 44 million people across the globe have Alzheimer or a related dementia and only 1 person in every four people have been diagnosed with AD [1]. As per statistics in the report [2], the global cost of dementia would reach $\$ 1$ trillion dollars by 2018 and will get increased to $\$ 2$ trillion by 2030 . Soft computing techniques have been promising out as medical expert systems for assisting medical practitioners for accurate diagnosis of the said disease. The main objective of this study is to evaluate the predictive performance of two alternative forecasting techniques that are Subtractive Clustering based Adaptive Neuro-Fuzzy Inference System (SCANFIS) with Fuzzy CMeans based ANFIS model (FCMANFIS) to reveal the most accurate forecasting technique for diagnosis of Alzheimer disease.

\section{LITERATURE REVIEW}

A significant research is being done in the domain of medical diagnosis using Neuro-fuzzy approach to diagnose various medical disorders. The literature related with the proposed study has been done and a general review of the same is presented as under:

A neuro fuzzy system based on fuzzy c-means (FCM) has been developed by Mohd Fauzi bin Othmanet al. [3] for diagnosis of diabetes. The suitability and performance of the FCM classification technique with respect to conventional Sub-clustering algorithm as a classifier in neuro fuzzy model ANFIS was evaluated for diagnosing diabetes on the dataset of 6172 records. This diagnostic system further optimized using weka and it achieved better accuracy as compared to the conventional approach. $K$. Arthi et al. [4] presented an automated intelligent diagnostic tool for diagnosis of autism using neuro-fuzzy approach cascaded with back propagation learning algorithm. The experimental results predicted the accuracy of $85-90 \%$ for this classifier.

Obi J.C.et al. [5] developed a neuro-fuzzy model for diagnosis of Alzheimer on the basis of neuropsychological tests. Nine symptoms like memory loss, difficulty in performing familiar tasks, problem with abstract thinking, etc. for $\mathrm{AD}$ have been taken as input

\footnotetext{
Manuscript received April 30, 2019; Accepted May26, 2019. (ID No. JMIS-19M-05-016)

Corresponding Author (*): Haneet Kour, Department of Computer Science and IT, University of Jammu, India, haneetkour9@gmail.com.

${ }^{2}$ Department of Computer Science \& IT,Bhaderwah Campus, University of Jammu, India, manhas.jatinder@gmail.com ${ }^{3}$ Department of Computer Science and IT, University of Jammu, India, vinodsharma@ jammuuniversity.in
} 
parameters each with three input linguistic values (low, medium and high) and one output.

R. Sampath et al. [6] implemented a hybrid Neuro-Fuzzy Runge Kutta (HNFRK) Classifier to predict Alzheimer disease from the data set of 150 fMRI images consisting of $95 \mathrm{AD}$ and 55 healthy controls samples. The proposed approach started with preprocessing the fMRI images using histogram based thresholding approach and then normalization of preprocessed image to MNI standard using SPM2 has been performed. Parcellation of brain scan into 8 ROI using spatial FCM algorithm, feature extraction and classification of features by ANFIS and Runge Kutta learning algorithm have been done. The performance of the proposed diagnostic system is evaluated with SVM and ANFIS classifier and proposed approach found to be better than the other two classifiers. K. Ashish et al. [7] presented a diagnostic system based on NFS and genetic algorithm for evaluation of depression by training the model with 312 data samples and validated with 10 data samples.

An ANN and Fuzzy logic based model has been developed using a dataset of 3032 data records by $L$. Andriuset al. [8] to evaluate Huntington disorder symptoms. This diagnostic system consists of four components for dataset formation, ANN prediction model to predict the functional capacity level, Mamdani fuzzy logic expert system determine a stage of reaction and decision module for determination of person's condition.

\section{METHODOLOGY}

For evaluating the performance of SCANFIS and FCMANFIS in diagnosis of $\mathrm{AD}$ disorder, the dataset for the study has been taken from Kaggle [9]. The data is in CSV format consisting of 336 instances (146-Diseased, 190-Normal) of the patients over the age group 60-98 years. The data acquired pre-processed with generalized methods and also normalized with different statistical methods. The dataset parameters are presented in the Table 1.

To attain the objective of the study, the experiments in Matlab 2018a have been performed. The data set for the study has been divided in the ratio of 60:20:20 (i.e. 202 samples for train data, 67 each for validation and test data) for two approaches. Three measures such as root mean square error (RMSE), prediction accuracy and precision have been calculated for these two models. Finally, the performances of these two approaches have been evaluated.

\begin{tabular}{ll}
\hline Attribute & Meaning \\
\hline Subject ID & Subject Identification \\
M/F & Gender of the patient \\
MMSE & Mini Mental State Examination \\
EDUC & Years of Education \\
SES & Socioeconomic Status \\
eTIV & Estimated total intracranial volume \\
CDR & Clinical Dementia Rating \\
Age & Age of patient \\
nWBV & Normalize Whole Brain Volume \\
ASF & Atlas Scaling Factor \\
Group & Normal / Diseased \\
\hline
\end{tabular}

In the first phase of the study, the SCANFIS [10, 11] based $\mathrm{AD}$ diagnostic system has been implemented with parameters presented in the Table 2. In the second phase, the diagnostic system implemented using FCMANFIS [11] with parameters presented in Table 3 .

Table 2: SCANFIS Parameters.

\begin{tabular}{ll}
\hline Parameter & Value \\
\hline Input Membership Function & Gauss \\
Output Membership Function & Linear \\
Learning Rule & Back propagation andleast \\
Epochs & square method \\
De-fuzzification Rule & 50 \\
Input and Output & Wtaver \\
No. of fuzzy rules & $9 * 1$ \\
No. of nodes & 33 \\
Reject Ratio & 672 \\
Range of Influence & 0.15 \\
Squash Factor & 0.5 \\
Accept Ratio & 1.25 \\
\hline
\end{tabular}

Table 3: FCMANFIS Parameters.

\begin{tabular}{lll}
\hline Parameter & Value & \\
\hline Input Membership Function & Gauss & \\
Output Membership Function & Linear & propagation \\
Learning Rule & Back andleast square method \\
Epochs & 60 & Wtaver \\
De-fuzzification Rule & 9 & 1 \\
Input & 15 \\
Output & 512 \\
No. of fuzzy rules & \\
No. of nodes & \\
\hline
\end{tabular}

\section{RESULT}

After performing the experiments, it has been found out 
that these two techniques can diagnose $\mathrm{AD}$ but FCMANFIS predicted AD efficiently than SCANFIS model. The generated SCANFIS and FCMANFIS models are represented in Fig. 1 and Fig. 2 respectively. The FCMANFIS predicted accuracy of $99.10 \%$ with RMSE of 0.17126 and $100 \%$ precision whereas SCANFIS predicted accuracy of $95.83 \%$ with RMSE of 0.3549 and $99.2 \%$ precision respectively. The experimental results are presented in Table 4 and Table 5. Also, F-score predicted by SCANFIS and FCMANFIS models are 0.9497 and 0.9895, thus FCM based ANFIS outperformed SCANFIS model.

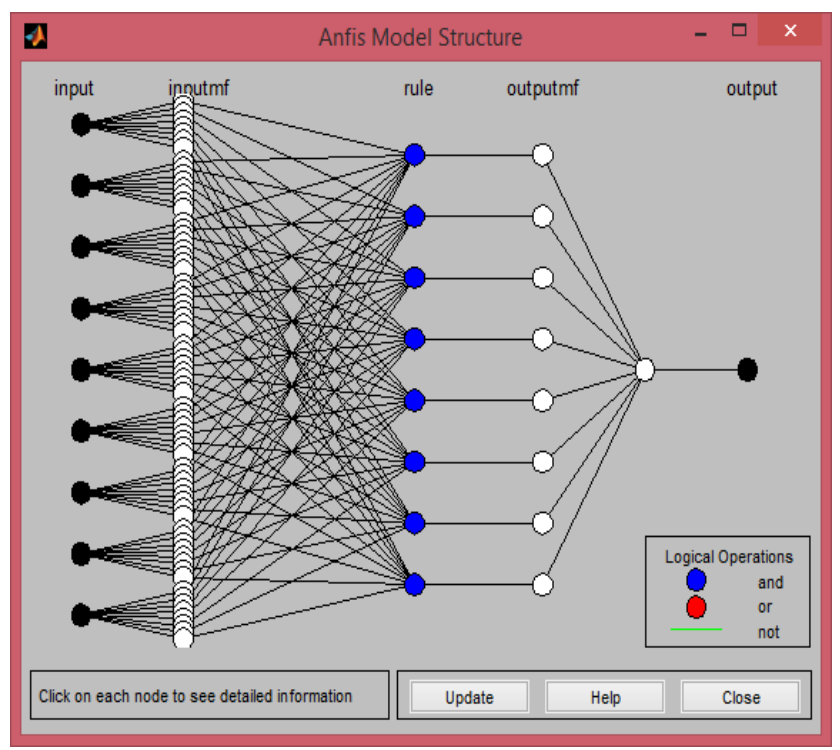

Fig. 1. SCANFIS generated model.

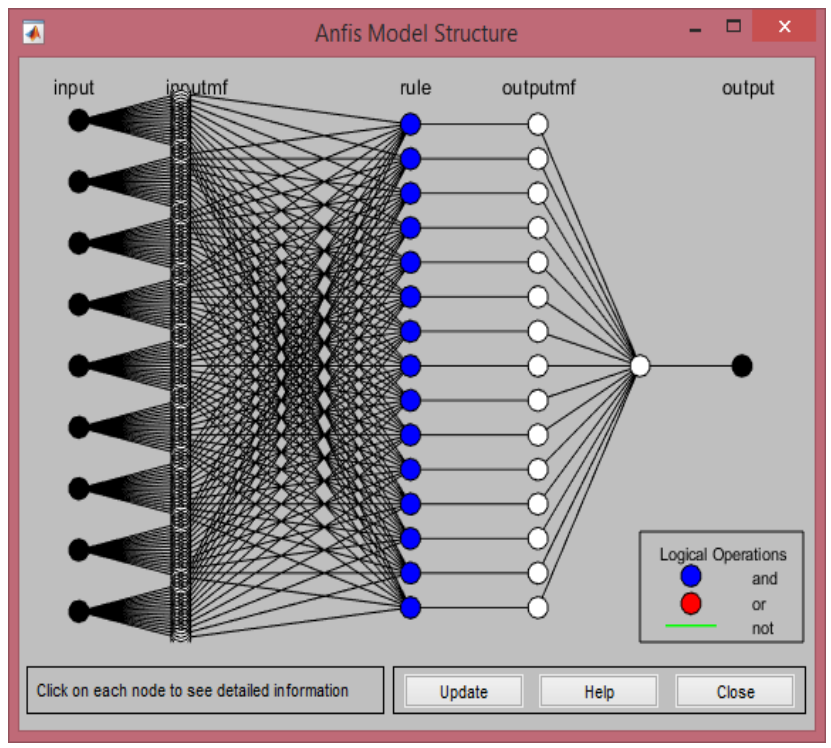

Fig. 2. FCMANFIS generated model.
Table 4: Evaluation of SCANFIS with FCMANFIS

\begin{tabular}{lll}
\hline Parameter & SCANFIS & FCMANFIS \\
\hline Accuracy & $95.83 \%$ & $99.10 \%$ \\
Precision & $99.2 \%$ & $100 \%$ \\
F-Score & 0.9497 & 0.9895 \\
\hline
\end{tabular}

Table 5: Error (in RMSE).

\begin{tabular}{lll}
\hline Error & SCANFIS & FCMANFIS \\
\hline Training Error & $1.82 * \mathrm{e}-04$ & $4.19 * \mathrm{e}-05$ \\
Validation Error & 0.23246 & 0.1865 \\
Testing Error & 0.3549 & 0.17126 \\
\hline
\end{tabular}

\section{CONCLUSION}

The experimental results show that SCANFIS and FCMANFIS can be efficiently used as expert systems for medical diagnosis. The performance of FCMANFIS is found to be better than that of SCANFIS in terms of accuracy, RMSE and precision. In the present comparative study, binary classification problem was taken. This study will be further enhanced to multi-class problem for comparative purposes.

\section{REFERENCES}

[1]. 2017 Alzheimer's

Statistics, https://www.alzheimers.net/resources/alzheimersstatistics/, 2018.

[2]. Cost and projections in the UK and globally, https://www.Dementiastatistics.org/statistics/cstand-projections-in-the-uk-and-globally/,2018.

[3]. M. F. Othman and T. M. Yau, "Neuro Fuzzy Classification and Detection Technique for Bioinformatics Problems", in Proceedings of the IEEE First Asia International Conference on Modelling \& Simulation, 2007

[4]. K. Arthi and A. Tamilarasi, "Prediction of autistic disorder using neuro-fuzzy system by applying ANN technique", International Journal of Developmental Neuroscience, vol. 26, no. 7, pp. 699-704, 2008.

[5]. J. C. Obi and A. A. Imainvan, "Decision Support System for the Intelligent Identification of Alzheimer using Neuro Fuzzy logic", International Journal on Soft Computing, vol. 2, no. 2, pp. 25-38, 2011.

[6]. R. Sampath and A. Saradha, "Alzheimer's Disease Classification Using Hybrid Neuro Fuzzy Runge-Kutta (HNFRK) Classifier", Research 
Journal of Applied Sciences, Engineering and Technology, vol. 10, no. 1, pp. 29-34, 2015.

[7]. K. Ashish, A. Dasari, S. Chattopadhyay and N. B.Hui, "Genetic-neuro-fuzzy system for grading depression", Applied Computing and Informatics, vol. 14, no. 1, pp. 98-105, 2018.

[8]. L. Andrius, M. R.Maskeliūnas and D. Robertas, "ANN and Fuzzy Logic Based Model to Evaluate Huntington Disease Symptoms", Hindawi Journal of Healthcare Engineering, Vol. $1,2018$.

[9]. Detecting Early Alzheimer's using MRI Data and Machine Learning,https://www.kaggle.com, 2019.

[10]. M. M. Gupta, "Fuzzy Logic and Neural Networks", IEEE Trans. Neural Networks, vol. 3, no. 5, pp. 636-639, 1992.

[11]. Subtractive Clustering, https://www.mathworks.com/help/fuzzy/subclust. html, 2019 [online].

[12]. FCM, http://www. mathworks.in/help/fuzzy/fcm. html, 2019 [online]. 